Revue musicale OICRM

\title{
Le Conservatoire de Paris et la médiation de la musique. Résistances et développements
}

\section{Lucie Kayas}

Volume 7, numéro 2, 2020

URI : https://id.erudit.org/iderudit/1072416ar

DOI : https://doi.org/10.7202/1072416ar

Aller au sommaire du numéro

Éditeur(s)

Observatoire interdisciplinaire de création et recherche en musique (OICRM)

ISSN

2368-7061 (numérique)

Découvrir la revue

Citer cet article

Kayas, L. (2020). Le Conservatoire de Paris et la médiation de la musique. Résistances et développements. Revue musicale OICRM, 7(2), 78-91.

https://doi.org/10.7202/1072416ar
Résumé de l'article

Établissement d'enseignement supérieur de la musique, le Conservatoire de Paris (CNSMDP) a récemment intégré la médiation à la formation tant des interprètes que des musicologues. Après avoir exploré le cadre institutionnel dans lequel s'est inséré cet enseignement et retracé l'histoire des débuts de cette discipline dans ce lieu, cet article s'interroge sur les résistances rencontrées par la médiation de la musique au sein du CNSMDP. 


\title{
Le Conservatoire de Paris et la médiation de la musique. Résistances et développements
}

\author{
Lucie Kayas
}

\begin{abstract}
Résumé
Établissement d'enseignement supérieur de la musique, le Conservatoire de Paris (CNSMDP) a récemment intégré la médiation à la formation tant des interprètes que des musicologues. Après avoir exploré le cadre institutionnel dans lequel s'est inséré cet enseignement et retracé l'histoire des débuts de cette discipline dans ce lieu, cet article s'interroge sur les résistances rencontrées par la médiation de la musique au sein du CNSMDP.

Mots clés : Conservatoire de Paris ; élitisme ; enseignement ; histoire ; médiation de la musique.
\end{abstract}

\begin{abstract}
An institution of higher learning for music, the Conservatoire de Paris (CNSMDP) recently added mediation into performers and musicologists' training. After exploring the institutional framework in which this discipline was introduced and after retracing the history of the beginnings of mediation at CNSMDP, this article questions the resistance encountered by music mediation within the institution.
\end{abstract}

Keywords: Conservatoire de Paris; education; elitism; history; music mediation. 
Lieu dévolu principalement à la formation des interprètes, le Conservatoire de Paris (CNSMDP) s'est engagé récemment dans l'enseignement de la médiation aux musicologues et aux musicien·ne·s se destinant à la pédagogie, ouvrant ainsi les cursus à des disciplines moins exclusivement musicales, tournées notamment vers la sociologie et l'étude des publics. Afin de comprendre en quoi ce choix est révélateur à la fois de l'évolution de la musicologie et de ses pratiques, et des résistances de l'institution à une médiation musicale qui s'imposerait aux interprètes et aux compositeurs, nous reviendrons sur l'histoire du Conservatoire et de ses missions afin d'y observer l'apparition de la médiation et d'en étudier les développements.

Cet article n'est pas le fait d'une théoricienne de la médiation, mais émane d'une musicologue ayant une expérience du terrain à la fois comme ancienne responsable d'un service éducatif ${ }^{1}$ et comme enseignante au Conservatoire ${ }^{2}$. Cette qualité de témoin ne lui confère donc pas la distance du chercheur. Cependant, un certain nombre d'entretiens ont été menés auprès d'une dizaine d'enseignant·e·s et de membres de l'équipe pédagogique et administrative du Conservatoire ${ }^{3}$ afin de recueillir leurs réflexions et leurs expériences. Cet article s'appuie également sur les travaux de Marie-Christine Bordeaux, Sylvie Pébrier et Louise Béguin qui seront détaillés par la suite.

L'hypothèse explorée ici serait que la nature élitiste de l'enseignement du CNSMDP serait un frein au développement de la médiation et à son inscription comme discipline à part entière dans cette institution.

\section{Le CONSERVATOIRE : UNE INSTITUTION CONSERVATRICE?}

Issu directement de la Révolution française, le Conservatoire est fondé en 1795. Il naît de la fusion entre l'École royale de chant et de déclamation (1783) et l'Institut national de musique (1793, émanation du Corps de musique de la garde révolutionnaire). En cela, il constitue une sorte de synthèse paradoxale entre les idéaux de l'Ancien Régime et ceux de la Révolution. Selon son premier directeur, Bernard Sarrette (1765-1858), le Conservatoire a pour mission de former les artistes nécessaires à la solennité des fêtes républicaines, au service militaire des nombreuses légions de la patrie ainsi qu'aux spectacles. Le Journal de Paris du 27 octobre 1796 reformule ainsi le projet : "La conservation de ce qu'il y a de plus pur et de beau dans l'art tel qu'il est, et la facilité de l'enrichir de ce qui lui manque " (ChassainDolliou 1995, 2e de couverture). Il s'agit donc à la fois de conserver le patrimoine musical - objectif facilité par la création de la bibliothèque et d'ateliers d'organo-

1 L'auteure a été responsable des actions éducatives du théâtre du Châtelet (Paris) de 2000 à 2012.

2 Professeure de la classe des Métiers de la culture musicale, autrefois Culture musicale, depuis septembre 2008.

3 Sabine Alexandre, responsable de la professionnalisation et de la médiation ; Liouba Bouscant, cheffe du département musicologie et analyse ; Philippe Brandeis, directeur des études musicales et de la recherche ; Claude Delangle, professeur de saxophone ; Sylvain Griotto, compositeur ; Claire-Marie Leguay, assistante de la classe de piano de Michel Dalberto ; Vincent Lê Quang, professeur d'improvisation générative ; Bruno Mantovani, ancien directeur (2010-1019); Alain Poirier, ancien directeur (2000-2009). 
logie, et de favoriser la création. "La plus précieuse uniformité » de l'enseignement (ibid., p. 19) est garantie par l'édition de multiples méthodes éditées en interne par l'imprimerie du magasin du Conservatoire.

Outre l'épisode sombre de la Restauration", deux déménagements notamment vont apporter leurs lots de modifications au projet initial. En 1911, le Conservatoire national de musique et d'art dramatique quitte la rue Bergère (près des Grands Boulevards) pour s'installer au numéro 14 de la rue de Madrid (à proximité de la gare Saint-Lazare). Mais c'est en 1946 que la scission intervient entre musique et théâtre, scission dont l'impact sur des disciplines comme le chant mériterait d'être étudié. La loi du 7 octobre 1946 instaure deux écoles distinctes : le Conservatoire national de musique et le Conservatoire national d'art dramatique, qui réintègre les locaux de la rue Bergère-rue du Conservatoire. Par la suite, le déménagement de 1990 voit le Conservatoire rejoindre le site excentré du parc de La Villette, devenant ainsi le voisin direct de la Cité de la musique (à laquelle s'adjoindra la Philharmonie de Paris en $2015^{5}$ ). Le Musée des instruments quitte alors le giron du Conservatoire pour intégrer celui de son nouveau voisin - la Cité de la musique -, devenant Musée de la musique.

$\mathrm{Au}$ fil du temps, le Conservatoire s'est enrichi de nouvelles classes, témoignant de son adaptation à l'évolution de la vie musicale (même si elle peut sembler lente, à témoin la création tardive de la classe de percussion en 1947) et d'ouverture allant au-delà de la musique classique : ouvrent successivement les classes de saxophone (1942), percussion (1947), nouvelles technologies (1984), improvisation générative (1992), accordéon (2002) et musique à l'image (2012), sans oublier l'apparition de départements, dont ceux de Jazz et musiques improvisées (1991), Pédagogie (1992), Musique ancienne (1984) et Formation supérieure aux métiers du son (1997). Notons également, et ce dans plusieurs disciplines, l'existence de classes différentes pour les hommes et les femmes jusqu'à la Deuxième Guerre mondiale, ce qui supposait des concours d'entrée et de sortie distincts (voir Ravet 2011, p. 68-82).

En 2018, soit quelque 220 ans après sa création, la mission du Conservatoire se voit redéfinie ainsi :

Le Conservatoire a pour mission principale de dispenser un enseignement hautement spécialisé et une formation professionnelle de haut niveau dans les domaines de la musique, de la danse et des nouvelles technologies du son.

Cet enseignement comprend les connaissances théoriques et la maîtrise pratique nécessaires à l'exercice de ces arts ou professions dans les activités suivantes : disciplines instrumentales classiques et contemporaines, musique ancienne, jazz

4 En France, la période de la Restauration (restauration du pouvoir monarchique) se situe entre 1814 et 1830, avec pour souverains successifs Louis XVIII et Charles X. Le Conservatoire fut supprimé le $1^{\text {er }}$ janvier 1816 et remplacé par l'École royale de musique et de déclamation ; il retrouvera son nom en 1822, sous la direction de Cherubini.

5 Inaugurée le 7 décembre 1995, la Cité de la musique s'est vue adjoindre la Philharmonie de Paris, devenant Établissement public à caractère industriel et commercial (EPIC), créé par un décret du 24 septembre 2015 : la Cité de la musique - Philharmonie de Paris. Il s'agit d'un un complexe musical combinant notamment quatre salles de concert, le Musée de la musique ainsi qu'une quinzaine de salles à vocation pédagogique. 
et musiques improvisées, disciplines vocales, écriture, composition, direction d'orchestre, musicologie et analyse, pédagogie et formation à l'enseignement, métiers du son, et, pour la danse, danse classique et danse contemporaine. La formation s'inscrit aujourd'hui dans le cadre de l'harmonisation européenne des cursus de l'enseignement supérieur (LMD). (Site Web du CNSMDP, onglet « L'école Missions $;$; c'est nous qui soulignons)

On est frappé par l'expression « formation professionnelle » qui vient comme contrebalancer «l'enseignement hautement spécialisé » que l'on peut comprendre comme une variante de l'excellence instrumentale ou musicologique. Il s'agit désormais de préparer aussi l'avenir professionnel des élèves, soit de s'interroger sur les métiers de musicien ne et de danseur'euse, sur leur place dans la cité - bref, d'adapter leur formation aux contraintes du milieu musical. La mention du LMD (LicenceMaster-Doctorat), soit l'inscription des diplômes dans le cadre du processus de Bologne ${ }^{6}$ et l'harmonisation européenne des cursus de l'enseignement supérieur, démontre cette fois une ouverture géographique visant à faciliter la mobilité des étudiant $\cdot e \cdot s$ et donc à leur procurer une formation non plus exclusivement française, mais ouverte au plan international.

La médiation de la musique intervient en tant que nouvelle composante du métier de musicien-ne. Peut-être faut-il préciser ici que c'est intentionnellement que l'expression «médiation de la musique » est préférée à celle de « médiation culturelle ». Dans un article de décembre 2008, Marie-Christine Bordeaux retrace les débuts de la médiation culturelle en France : "Il faut attendre 1997, année de lancement du programme gouvernemental "nouveaux services - emplois jeunes", pour que la médiation culturelle soit reconnue comme une fonction et un métier spécifiques " (Bordeaux 2008). Abordant ensuite « les résistances à la médiation dans le domaine culturel », Bordeaux mentionne les cas des musées, du théâtre et de la danse, mais omet la musique sans que l'on puisse comprendre pourquoi. Cette question clé réapparait dans la dernière partie du même article. L'auteure en vient à s'interroger sur l'existence d'un modèle de médiation culturelle généraliste :

Existe-t-il un modèle générique de médiation culturelle, qui serait la matrice de toutes les médiations observables dans les différents domaines artistiques et culturels ? Les conditions de possibilités de la médiation sont-elles de même nature dans les musées, la danse, le cinéma ? Si oui, nous pouvons continuer à parler de LA médiation culturelle, autrement que comme un ensemble à peu près homogène de techniques d'intervention, comparables d'un domaine à un autre. Sinon, comment construire l'articulation entre les différents modèles locaux de la médiation culturelle : médiation muséale, patrimoniale, théâtrale, etc., et sur quel critère discriminant? (ibid.)

$6 \quad$ Initié en 1998, ce processus vise à harmoniser les systèmes d'études supérieures des différents pays européens et à faciliter ainsi la mobilité des étudiant·e·s. En 1999, 29 pays ont signé l'accord de Bologne qui a connu différents prolongements. Ainsi, en 2010, la déclaration finale de la conférence de Budapest et Vienne a annoncé la création d'un Espace européen de l'enseignement supérieur. 
En choisissant d'intégrer la médiation en son sein, le Conservatoire affirme de facto que la médiation de la musique doit faire partie des compétences du ou de la musicien·ne, ce qui ne signifie pas pour autant qu'elle lui soit réservée. Dans ce cadre, les interprètes semblent les premiers concernés, mais très vite, les musicologues se sont trouvés plus particulièrement sollicités, sans doute du fait de leur plus grande facilité à parler de la musique et de la transformation de la classe de Culture musicale en Métiers de la culture musicale, sur laquelle on reviendra plus tard. Ces deux types d'élèves incarnent en quelque sorte deux rapports à la musique : le rapport sensible et le rapport intelligible dont la combinaison génère le plaisir esthétique, selon Liouba Bouscant (entrevue avec l'auteure, 18 février 2019). Ceci pose la question de l'identité, du profil du ou de la médiateur-rice de la musique : est-ce un·e généraliste, un·e spécialiste, un·e interprète, un·e compositeur·rice, un·e musicologue ? Consulté en février 2019, Bruno Mantovani s'exprimait ainsi :

Pourles musicologues, la médiation estl'essence du métier. Pour les futurs professeurs, c'est aussi une énorme partie de ce qui les attend. Pour les compositeurs, c'est une condition de survie. Pour les instrumentistes, tout dépend de leur voie, mais tout ce qui a été monté avec la Cité s'adresse en priorité à eux. (Courriel personnel adressé à l'auteure, 25 février 2019)

Afin de mieux comprendre la mise en place de la médiation au Conservatoire, il n'est pas inutile de prendre conscience de l'organisation de l'institution et de la pédagogie en fonction de la notion de classe : la classe de piano, la classe d'histoire de la musique...

Sur 1200 élèves en 2019, on compte environ 650 interprètes, 180 compositeur rice·s et élèves en écriture, 10 élèves de la classe de Métiers de la culture musicale sur 80 élèves du département Musicologie et analyse, et 80 en pédagogie. Ce serait ignorer les quelque 200 danseur euse's qui travaillent souvent avec les jeunes compositeur'rice's ou avec des instrumentistes interprétant un répertoire contemporain. N'oublions pas les enseignant·e's qui sont nombreux'euses à pratiquer eux'ellesmêmes différents types de médiation. Autrement dit, la population concernée par la médiation, répartie en neuf départements ${ }^{7}$, se chiffre en centaines.

L'organisation des cours par classe constitue depuis son origine l'une des spécificités de l'enseignement du Conservatoire (voir Campos 2016, p. 45-52). Pour se définir, un·e pianiste, par exemple, ne dira pas seulement «j'ai un premier prix du CNSM de Paris ", mais précisera dans quelle classe : chez Roger Muraro, chez Frank Braley... Cette verticalité se prolonge dans les départements qui sont comme des silos indépendants luttant pour se mettre en synergie. Sans doute la nature pluridisciplinaire de la médiation - qui impliquerait plutôt une certaine horizontalité - se heurte-t-elle à ce fonctionnement.

Il faut encore préciser quelles sont les classes concernées dans le département Musicologie et analyse. Elles sont présentées par ordre de leur création, qui s'étale sur plus de 120 ans. Fondée en 1871 à l'initiative du nouveau directeur de l'époque,

$7 \quad$ Danse, Disciplines instrumentales, Disciplines vocales, Écriture et composition, Jazz et musiques improvisées, Musicologie et analyse, Musique ancienne, Métiers du son, Pédagogie. 
Ambroise Thomas, la classe d'Histoire de la musique est de loin la plus ancienne. Elle ne s'imposera véritablement qu'avec l'arrivée de Louis-Albert Bourgault-Ducoudray en 1878, qui fixe les orientations de son enseignement lors de sa leçon inaugurale ${ }^{8}$ :

C'est de l'examen réfléchi du passé, c'est de la connaissance raisonnée de la tradition que découle la vraie liberté, née du progrès légitime et de la saine originalité. [...] Le véritable interprète doit s'identifier aussi profondément que possible avec la pensée du compositeur ; pour cela son intelligence doit embrasser dans leur ensemble toutes les parties de l'œuvre à l'exécution de laquelle il doit concourir. (Bourgault-Ducoudray 1878, p. 2)

Notons qu'à cette époque, de 1878 à 1909, cette classe s'adresse aux interprètes (instrumentistes et chanteur·se's) et compositeur-rice's ou élèves en écriture (et non à des élèves spécialisés) qui participent aux exemples musicaux donnés en cours, le phonographe n'en étant qu'à ses débuts.

La classe d'analyse apparaît en 1947 (la $2^{\mathrm{e}}$ dénomination de la classe de Messiaen de 1947 à 1974 étant « Analyse et esthétique musicale »), l'Esthétique s'en distinguant en 1956. Plus de 35 ans s'écoulent avant la création de la Classe de culture musicale en 1993, devenue classe des Métiers de la culture musicale en 2014. La discipline " culture musicale ", qui coexiste avec analyse, esthétique et histoire de la musique, émerge en France à l'incitation d'un rapport du Comité technique de l'enseignement musical qui affirmait que "l'accent devra être particulièrement mis sur la formation culturelle et pédagogique des futurs enseignants ». Dans cette logique, le Certificat d'aptitude à l'enseignement de la culture musicale $\left(\mathrm{CA}^{9}\right)$ est créé en 1987. Dans son Rapport concernant l'examen du premier CA de culture musicale, François Rossé s'exprime ainsi : " Il convient de redonner au terme de culture un sens actif en joignant à la connaissance historique et à l'approche esthétique les techniques de décodage (analyse) et un minimum de pratique vécue (pratique instrumentale et créative) " (cité dans Schneider 2000, p. 33). Comme le fait remarquer Corinne Schneider, le professeur de culture musicale est donc à la fois un généraliste et un spécialiste, ce à quoi l'on pourrait ajouter qu'il combine les compétences analytique, historique, esthétique et artistique (pratique instrumentale et créative) (ibid., p. 26). Le passage de la « culture » aux " métiers de la culture » en 2014 a résulté d'une réflexion sur la professionnalisation et sur les inévitables recouvrements entre les classes d'analyse, d'histoire et d'esthétique d'une part, et celle de culture musicale de l'autre.

Toutes ces classes, accessibles sur concours, s'adressent à des musicologues, ce qui n'empêche pas les interprètes de pouvoir s'y présenter, le fait devenant de plus en plus rare. Ainsi le Conservatoire se modernise, s'ouvre à d'autres disciplines, mais la médiation, qui ne s'inscrit pas exclusivement dans une classe spécifique, mais fait appel à des compétences transversales - y compris non musicales -, peine par là même à trouver sa place.

8 Voir Le Ménestrel, $1^{\text {er }}, 8,15$ et 22 décembre 1878. Voir également Campos 1995.

9 Le CA, diplôme national homologué par le Ministère de la culture, valide les connaissances et les compétences générales et professionnelles correspondant au niveau de fin de second cycle d'études supérieures préparant à l'enseignement de la musique. Ce diplôme de pédagogie et formation à l'enseignement de la musique confère aujourd'hui le grade de Master. 


\section{NÉCESSITÉ D’ENSEIGNER LA MÉDIATION AU CONSERVATOIRE}

Apparue dans le milieu culturel français au milieu des années 1990, la médiation musicale, au même titre que la médiation culturelle, offre de nouveaux développements à la politique de démocratie culturelle mise en place par le gouvernement français après 1981. Un temps de latence a séparé cette prise de conscience politique de la reconnaissance d'une nécessité de former les acteurs de la médiation, qu'ils soient ou non interprètes, et force est de constater que le Conservatoire a pris un certain retard par rapport aux universités.

Ainsi le premier département de Médiation culturelle a été créé en France en 1994 à la Sorbonne Nouvelle. Il faut attendre 2007 pour l'ouverture d'une option "Médiation musicale " au sein du Master 2 Musique et Musicologie de ParisSorbonne, à l'initiative de l'ONDIF (Orchestre national d'Île-de-France). En 2014 s'ouvre le Master 2 « Médiation de la musique » à Paris-Sorbonne et Sorbonne Nouvelle, suivi du Master "Médiation de la musique " (formation complète en deux ans : master 1 et master 2) en 2019 à Sorbonne Université (ex Paris-Sorbonne) et Sorbonne Nouvelle ${ }^{10}$. Les dénominations " médiation musicale " et " médiation de la musique " laissent penser qu'une formation spécifique à la médiation de la musique a un sens, alors que prédominaient jusque-là les formations généralistes à la médiation culturelle. Le Conservatoire s'inscrit dans cette perspective d'une spécialisation musicale. Dans son rapport de 2015 consacré aux publics de la musique classique, Sylvie Pébrier aborde la question de la formation à la médiation de la musique dans les établissements d'enseignement supérieur français et remarque la regrettable hétérogénéité de l'offre pédagogique en la matière (voir Pébrier 2015, p. 219 sq).

$\mathrm{Au}$ Conservatoire de Paris, les premières expériences de médiation datent de 2002, l'établissement étant alors placé sous la direction d'Alain Poirier ${ }^{11}$. C'est à son initiative que trois premières activités de médiation ont eu lieu : deux en prison, la troisième en hôpital pour enfants. Dans les trois cas, il s'agissait d'" un répertoire simple et abordable, précédé de présentation rapide des instruments et du programme, et suivi de questions de la part du public » (courriel personnel à l'auteure, 25 février 2019). Le choix des formations n'est sans doute pas anodin. Pour la prison des hommes de Fleury-Mérogis, un ensemble de jazz avait été retenu tandis que pour le quartier des femmes de la même prison, un ensemble à cordes avait été choisi, une répartition par genre qui mériterait que l'on s'y attarde : jazz pour les hommes, cordes pour les femmes. Dans le cas de l'hôpital Necker-Enfants malades (Paris), un trio d'anches s'était déplacé. Pour les trois concerts, le directeur du Conservatoire avait accompagné les élèves, mais nous n'en savons pas plus sur la manière dont ces concerts avaient été ou non préparés. Restent les souvenirs du directeur de l'époque : «Expériences riches, comme tu l'imagines, ayant laissé des traces chez

10 Je remercie Cécile Prévost-Thomas (maîtresse de conférence Département de Médiation Culturelle - UFR Arts et Médias de l'Université Sorbonne Nouvelle) de m'avoir fourni ces informations.

11 Musicologue, ancien directeur du CNSMDP (2000-2009), Alain Poirier (né en 1954) est actuellement directeur de la recherche au CNSM de Lyon. 
les musicien·ne's qui y ont participé face à un public inhabituel » pour ce qui est de Fleury-Mérogis, " expérience beaucoup plus forte encore face à des enfants parfois très atteints de leucémie, amputation, etc. qui a été bouleversante pour les trois musiciens et moi » (courriel personnel à l'auteure, 25 février 2019) quant à celle de l'hôpital Necker-Enfants malades. Remarquons encore que, dans les trois cas, les médiateur'rice's se confondaient avec les interprètes et que la formule choisie était celle du concert présenté.

Une proposition de formation a suivi de peu ces expériences, initiative venue du théâtre du Châtelet dont la responsable des actions pédagogiques ${ }^{12}$, ayant découvert les ateliers de création proposés par plusieurs théâtres d'opéra britanniques, cherchait des artistes français susceptibles de mener ce type d'action sans faire appel à des artistes-animateurs britanniques. La question est devenue alors : comment former de jeunes musiciens français à ce type de pratique? Consciente que la Guildhall School of Music de Londres préparait les artistes à ces activités, elle a choisi de s'adresser au lieu d'excellence de formation des musiciens en France : le Conservatoire de Paris (CNSMDP ; élèves des classes de percussion et de saxophone). Le projet associait le théâtre du Châtelet, le CNSMDP ainsi qu'une classe du collège Dorgelès (Paris 18 ${ }^{\mathrm{e}}$ ) et son professeur de musique qui animait un atelier d'informatique musicale. Il s'est déroulé en trois temps, d'octobre 2002 à mars 2003 : tout d'abord un atelier pour les élèves du Conservatoire qui ont fait eux-mêmes l'expérience de la création collective sous la direction de Mark Withers (animateur musical issu de la Guildhall), puis une observation de la classe de collège, et enfin un travail avec les collégien·ne·s invité·e·s à créer une pièce à partir des procédés présentés par les étudiant· $\mathrm{e} \cdot \mathrm{s}$. Si le déroulement de ce module expérimental a été satisfaisant, tant du point de vue des élèves que de celui des organisateurs, force est de constater qu'il n'a pas été renouvelé, le Conservatoire n'ayant pas souhaité s'engager financièrement et le Châtelet refusant prendre en charge tous les frais. Quand on interroge l'actuel directeur des études du CNSMDP, déjà en poste à l'époque, on comprend que les réticences étaient liées notamment à l'organisation des études : le DNSPM (Diplôme national supérieur professionnel de musicien) était conçu d'un bloc et avait pour but l'excellence instrumentale. Ce n'est finalement qu'avec le passage au LMD (septembre 2008), structurant le cursus en un premier et un second cycles intégrant réflexion, recherche, interrogations sur sa propre pratique et rapport aux publics, qu'une place s'est dessinée pour la médiation ${ }^{13}$.

\section{FORMER À LA MÉDIATION}

Quinze ans plus tard, la situation a changé et quatre dispositifs participent de la formation à la médiation au CNSMDP : l'Initiation à la médiation, la Classe des métiers de la culture musicale, la Méthodologie pratique de recherche et de la médiation et le Séminaire médiation. Pour autant, certaines réticences demeurent et d'autres difficultés sont apparues.

13 Selon un entretien personnel avec Philippe Brandeis, janvier 2019. 
Posons comme préalable une définition large de la médiation musicale que nous concevons comme toute action visant à rapprocher un public d'une musique. Elle induit que la formation à la médiation va se développer sur trois plans : la connaissance des publics, celle des répertoires et celle des modalités de la médiation (dispositif mis en place en partenariat avec des lieux précis à destination de publics ciblés, stratégie adoptée, approches choisies...). Née d'une sollicitation du service pédagogique de la Cité de la musique $^{14}$, la première formation à la médiation s'est inscrite sous forme d'expérience pilote au cours de l'année scolaire 2009-2010 (voir Béguin ${ }^{15}$ 2017, p. 34-35). Elle est depuis placée sous la responsabilité de Sabine Alexandre (responable professionnalisation et médiation). En quête de futurs artistes médiateur·rice·s, la Cité de la musique avait pris en charge l'ensemble des coûts d'une formation destinée aux interprètes volontaires et ceux de l'organisation des concerts. On constate donc qu'une fois encore, comme dans le cas du Châtelet, le Conservatoire a été motivé par une sollicitation extérieure émanant du milieu musical. Il s'agissait d'une formation des interprètes, amené·e·s à présenter des concerts symphoniques donnés par eux·elles-mêmes à la Cité de la musique. Le projet était alors d'initier tou·te·s les étudiant·e.s volontaires (quel qu'ait été le département auquel ils et elles étaient rattaché·e·s) uniquement par la pratique, autour de deux coproductions de la Cité de la musique et du Conservatoire : Abramaderna!-concert éducatif autour de la pièce de Pierre Boulez Rituel in memoriam Maderna ${ }^{16}$ - et Les noces de Figaro de Mozart. Dans la convention liant les deux établissements, les objectifs étaient libellés comme suit :

Les sessions de formation viseront à aider les étudiants à :

- Prendre conscience de la diversité des publics et des enjeux de la médiation culturelle,

- Acquérir des outils permettant d'échanger avec son public,

- Maîtriser des techniques pour appréhender les pièces du répertoire avec un public non spécialiste lors d'ateliers de préparation au concert.

Le public visé était un public scolaire venant assister aux spectacles et la formule choisie celle d'ateliers de préparation en classe. Toutes les sessions étaient animées par Sarah Goldfarb ${ }^{17}$.

En 2010, la réussite de ce module pilote amène sa transformation en DCO (discipline complémentaire optionnelle) ouverte à tous. À cette date, la formation, intitulée "Initiation à la médiation musicale en milieu scolaire », reste entièrement

14 La responsable en est Marie-Hélène Serra, depuis 2002.

15 Le mémoire de master de Louise Béguin constitue une étude de cas de l'enseignement de la médiation au CNSMDP à partir d'un stage réalisé dans l'établissement en 2016-2017.

16 Concert du 23 janvier 2010 dirigé par Pascal Rophé, mis en scène par Emmanuelle Cordoliani avec Victor Duclos, comédien-danseur.

17 Flûtiste belge diplômée des Conservatoires de Bruxelles et de Liège, Sarah Goldfarb poursuit ses études à la Guildhall School of Music de Londres où elle obtient un Master en « music performance and communication skills » puis un Master en composition musicale à la City University de Londres. 
financée par la Cité de la musique ${ }^{18}$. Son histoire peut se décliner sur trois plans : celui de l'évolution du public d'élèves auquel elle s'adresse, celui de la recherche d'un équilibre entre pratique et théorie - une théorie absente de la formule initiale, mais qui apparaît dès l'année scolaire 2010-2011 - et celui des champs d'application (scolaire, médiathèque, hospitalier...) ou des publics visés.

Pour ce qui est des étudiants concernés, on passe progressivement d'une formation ouverte à tou'te's à une formation obligatoire pour deux types d'élèves du Conservatoire en 2014 : les interprètes préparant le Diplôme d'État d'enseignement en conservatoire (DE) ${ }^{19}$ et les musicologues de la classe des Métiers de la culture musicale ${ }^{20}$. Se dessine alors un double questionnement : celui de l'articulation entre médiation et enseignement d'une part, et entre médiation et musicologie de l'autre. Le référentiel $\mathrm{du} \mathrm{DE}^{21}$ s'articule en deux grandes parties, la première concernant l'enseignement proprement dit, la seconde le fait d' " être acteur du projet d'établissement " dans lequel on enseigne. C'est cette dernière qui recouvre certains champs de compétences de la médiation, notamment la prise de parole en public, la maîtrise de la dynamique de groupe et la connaissance des territoires et de leurs équipements, à tel point qu'au CNSMDP, l'obligation de suivre la Formation à la médiation remplace le stage initialement prévu en conservatoire. Inversement, certains outils de l'apprentissage de la musique (apprentissage collectif d'un chant, pratique de l'improvisation...) participent des outils de la médiation : c'est le public auquel on s'adresse qui est différent, ainsi que le cadre et les objectifs. Dans le cas de la médiation, il s'agit plus du partage d'une pratique et d'un savoir ne donnant pas lieu à une évaluation des acquis, ce qui n'empêche pas un certain degré d'exigence dans les réalisations proposées par exemple au cours d'ateliers.

De leur côté, les élèves musicologues de la classe de Métiers de la culture musicale maîtrisent mieux le discours et la réflexion sur la musique que leurs camarades interprètes ; leur culture musicale est plus large, ce qui ne signifie pas pour autant qu'ils excellent dans la dimension orale de sa transmission ou la connaissance des publics. Dans l'optique d'une musicologue appliquée, cette classe propose de former les élèves

18 Cette répartition des charges a évolué à partir de 2012 pour atteindre aujourd'hui un équilibre entre la Cité de la musique - Philharmonie de Paris et le CNSMDP.

19 «Le DE est le diplôme le plus répandu pour l'enseignement de la musique instrumentale ou vocale dans les conservatoires. Toutes les disciplines et les esthétiques sont représentées : musique classique, jazz, musiques actuelles et musiques traditionnelles. Il s'obtient après une formation initiale dans les Pôles supérieurs d'enseignement artistique ou les Cefedem (Centres de formation des enseignants de musique et de danse), par le biais de la formation professionnelle continue ou de la validation des acquis de l'expérience. Le DE permet notamment de se présenter aux concours d'assistant territorial d'enseignement artistique de la fonction publique territoriale » (https://metiers.philharmoniedeparis.fr, consulté le 23 octobre 2019).

20 Comme précisé précédemment, cette classe résulte de l'évolution de la classe de Culture musicale en classe des Métiers de la culture musicale en 2014.

21 Ce référentiel est paru dans l'annexe I de l'arrêté du 29 juillet 2016 relatif au certificat d'aptitude et aux fonctions de professeur de musique et fixant les conditions de l'habilitation des établissements d'enseignement supérieur à délivrer ce diplôme, soit dans le Bulletin officiel no $261 \mathrm{du}$ ministère de la Culture d'août 2016 (voir https://www.culture.gouv.fr/Espace-documentation/Bulletin-officiel/Bulletin-officiel$\underline{\text { n-261-aout-2016, }}$ consulté le 10 juillet 2020). 
à différentes activités professionnelles : radiophonie, critique musicale, rédaction de notes de programme, etc., ainsi que la médiation. La question de la transmission se trouve donc au cœur des préoccupations de ses élèves. Se pose pour eux la question $\mathrm{du}$ rapport à leur propre pratique musicale ${ }^{22}$ : n'étant pas des instrumentistes adoubés par le concours d'entrée au CNSM (à la différence de leurs camarades interprètes), mais sélectionnés en musicologie, s'autorisent-ils l'utilisation de leur instrument dans le contexte élitiste du Conservatoire de Paris ? Cette question de l'élitisme occupe une place clé dans les représentations que les élèves se font d'eux-mêmes et de l'institution.

Le fait que le Conservatoire soit accessible sur concours place l'élitisme au centre de son enseignement depuis sa création. Dans son ouvrage Le Conservatoire de Paris et son histoire, Rémy Campos résume ainsi ce qui demeure de deux siècles d'histoire de l'institution : " Le concours d'abord et la doctrine républicaine qu'il met en œuvre. La classe ensuite et l'esprit de corps qu'elle engendre [...] Une certaine idée de la musique enfin qui valorise la virtuosité et la prestation soliste et dont la maîtrise de la lecture solfégique avait constitué le parangon. »(Campos 2016, p. 78-79). Comprenons que cet élitisme républicain (qui permet à chacun de réussir selon ses mérites) imprègne plus ou moins consciemment l'esprit des élèves d'aujourd'hui. Or, sélectionner l'élite revient à exclure les autres, ceux qui ne sont pas assez performants, tandis que la médiation développe un processus inclusif : toute personne a des aptitudes à apprécier ou pratiquer la musique sur lesquelles vont s'appuyer les médiateur·rice·s. Il semble que cet élitisme vienne percuter de plein fouet la notion de partage implicite à la médiation ; les élèves se trouvent confrontés à une véritable difficulté : abandonner l'élitisme tout en cultivant une excellence (dans l'acte de médiation) appliquée au partage avec des personnes qui ne sont plus considérées comme des publics potentiels, mais des participants, alors qu'ils ont parfois l'impression de renoncer à leurs exigences musicales et intellectuelles, de verser dans la démagogie.

On constate que l'hétérogénéité des élèves de cette formation ne va pas sans poser de problème. L'évolution du contenu de la formation découle en partie de cette question. Après une expérimentation uniquement tournée vers la pratique, la formule de l'année 2010-2011 introduit une partie théorique confiée à des intervenants extérieurs :

- Table ronde sur le thème « La médiation dans les institutions musicales (2h);

- Conférence "Les enjeux sociaux de la médiation musicale » (3h), assurée par Sophie Maisonneuve ${ }^{23}$.

L'approche pratique concerne :

- Les techniques d'animation (2h),

- Les techniques d'adaptation au public (2h).

22 Tous les élèves du département Musicologie et analyse jouent d'un instrument, une épreuve instrumentale figurant au concours d'entrée.

23 Agrégée de musique et docteur en histoire, Sophie Maisonneuve est maîtresse de conférence à l'IUT de Paris V, LAHIC/IIAC (CNRS) et consacre ses travaux à une sociohistoire des modalités de production, de circulation et d'appropriation de la musique. 
Le dernier volet de la formation s'intitule "Application à un répertoire ». Les étudiants animent des ateliers en milieu scolaire en préparation au concert. Ils réalisent deux ou trois ateliers, chacun représentant $15 \mathrm{~h}$ de préparation encadrée, allant de la découverte de l'œuvre à l'animation de l'atelier en passant par sa conception et son test. Les trois plans de la médiation mis en avant précédemment se trouvent désormais représentés et appliqués à un cas précis : connaissance du public scolaire, modalité de la médiation en atelier et connaissance d'œuvres : cette année-là, la Sixième symphonie "Pathétique " de Tchaïkovski, Le sacre du printemps de Stravinsky et La flute enchantée de Mozart (nouvelle coproduction CNSMDP-Cité de la musique), le tout étant précédé d'une initiation aux enjeux (sociaux et institutionnels) de la médiation.

C'est cette formule qui est devenue le noyau de la formation, étendue progressivement jusqu'à la maquette ${ }^{24}$ cristallisant le caractère obligatoire de la discipline (discipline complémentaire obligatoire ou DCO, désormais intitulée Formation à la médiation) à partir de 2014. L'articulation se fait entre un tronc commun théorique, les ateliers et les présentations de concert. Deux formes de médiation sont donc toujours pratiquées : ateliers scolaires et présentations de concerts. Le tronc commun $(24 \mathrm{~h}$ pour les interprètes, $27 \mathrm{~h}$ pour les musicologues) rassemble enjeux de la médiation musicale, prise de parole en public et conception et animation d'une médiation. Les ateliers sont pensés par champ - scolaire, territorial, hospitalier -, champs qui se sont donc étendus depuis le début, alors qu'ils étaient focalisés sur le milieu scolaire. $\mathrm{Au}$ départ, ils n'étaient pas présentés d'un point de vue théorique, à l'exception du champ hospitalier, mais leur connaissance fait désormais partie du tronc commun, permettant à tous les élèves d'avoir des informations sur une grande variété de terrains d'application possibles pour la médiation. Chaque atelier représente $21,5 \mathrm{~h}$ de formation-réalisation, ceux se déroulant en champ hospitalier allant jusqu'à $30 \mathrm{~h}$ du fait de la nécessité d'une connaissance approfondie du secteur. La présentation de concert donne lieu à $21 \mathrm{~h}$ de formation.

$\mathrm{Au}$ fil du temps, le contenu du tronc commun a fait débat tant parmi les élèves que parmi les enseignants, notamment quant à certaines redondances par rapport à la classe des Métiers de la culture musicale et à certains manques, notamment d'ordre théorique. Une refonte de la maquette tenant mieux compte des besoins et des capacités du public hétérogène auquel la formation s'adresse a donc été mise en œuvre. L'examen attentif de cette maquette met en lumière un troisième élément de ce dispositif, le séminaire médiation qui représente la dimension de la recherche, fondamentale dans le cadre du master. Organisé par le département de Musicologie et analyse ${ }^{25}$, il comporte trois sessions par an, chacune étant confiée à un professeur du département (Florence Badol-Bertrand, Lucie Kayas, Sylvie Pébrier) qui en propose la thématique : Formes de la médiation, Médiation et diversité culturelle, Médiation musicale en prison, Médiation et création, etc. L'invitation de personnalités extérieures, théoricien $\cdot n e \cdot s$ ou acteur·rice.s de terrain, la sollicitation des étudiant $\cdot \mathrm{e} \cdot \mathrm{s}$

25 Il a été initié par la responsable du département, Liouba Bouscant, en 2017-2018. 
pour présenter des cas personnels ou des études de cas font de ce séminaire un lieu de réflexion partagée entre enseignant·e·s, spécialistes, artistes engagé·e·s dans la médiation et étudiant·e·s. Précisons que ce séminaire est précédé de trois cours propédeutiques (Sylvie Pébrier) visant à donner aux étudiants les notions préalables nécessaires concernant les politiques culturelles et la connaissance des publics. Par ailleurs, le cours de Méthodologie pratique de recherche et de la médiation de Florence Badol-Bertrand (discipline complémentaire optionnelle) permet notamment aux étudiants de mener un projet de médiation intégralement, de la conception à la réalisation. Dans la même optique, le Conservatoire (et plus précisément les élèves de la classe des Métiers de la culture musicale) participe depuis 2018 à l'École d'hiver internationale en médiation de la musique, créée en 2017 par l'Université de Montréal, Paris-Sorbonne et Sorbonne Nouvelle - Paris 3, qui situe la réflexion au niveau de la francophonie.

L'enseignement de la médiation au Conservatoire de Paris a aujourd'hui dix ans. Ce choix de développer une formation à une médiation spécifiquement musicale constitue déjà une affirmation forte. On a pu en voir l'évolution et les atermoiements dans son déploiement auprès de deux types d'étudiant $\cdot e \cdot s$, musicologues et interprètes. Pour les premiers, la médiation se pose comme une branche de la musicologie qui serait moins théorique et ferait appel à des disciplines complémentaires issues de la sociologie et de la pédagogie, ouvrant également de nouveaux terrains de recherches. Pour les interprètes, elle est devenue partie intégrante du métier de musicien et à ce titre, devrait participer plus largement de leur formation et les concerner tout·e.s : instrumentistes, chanteur·euse's, chef·fe's de chœur et d'orchestre, mais également les danseur·euse's, en dehors champ musical. Autrement dit, la médiation, au même titre que la recherche, gagnerait à s'affranchir de la notion de «classe " pour se situer au confluent de toutes les disciplines en cultivant l'interdisciplinarité.

On peut également s'interroger sur les processus de médiation proposés, qui ont jusqu'à présent privilégié la présentation des œuvres dans l'optique d'un concert. En s'inspirant du modèle anglo-saxon, il serait possible d'initier les élèves à des formules créatives et participatives visant l'appropriation des phénomènes musicaux plus que la consommation du concert, dans l'optique d'une démocratie culturelle.

Il semble que les résistances à la médiation au sein du Conservatoire proviennent pour une part de cette dimension interdisciplinaire, qui se heurte dans un premier temps à l'exigence d'excellence qui caractérise le CNSMDP dans des disciplines spécifiques. Comment exceller à la fois dans la connaissance des répertoires, dans celle des publics et dans celle des techniques de médiation? L'autre point d'achoppement de situe dans le fonctionnement élitiste et vertical de la maison qui s'accommode mal de l'horizontalité de la médiation. Une réflexion sur la distinction entre élitisme et excellence permettrait peut-être de faire prendre conscience aux élèves et aux enseignants de cette ligne de crête fragile où se situe la médiation. 


\section{BIBLIOGRAPHIE}

Béguin, Louise (2017), La médiation de la musique au Conservatoire national supérieur de musique et de danse de Paris, mémoire de Master 2 de médiation de la musique, Université Sorbonne NouvelleParis 3.

Bordeaux, Marie-Christine (2008), «La médiation culturelle en France, conditions d'émergence, enjeux politiques et théoriques ", Culture pour tous. Actes du Colloque international sur la médiation culturelle, Montréal - Décembre 2008, https://www.culturesducoeur.org/Content/Docs Observatoire/86. PDF, consulté le 10 juillet 2020.

Bourgault-Ducoudray, L[ouis]-A[lbert] (1878), « Cours d'histoire générale de la musique, séance d'ouverture ", Le Ménestrel, $1^{\text {er }}$ décembre, $45^{\mathrm{e}}$ année, $\mathrm{n}^{\circ}$ 1, p. 1-3, https://gallica.bnf.fr/ark:/12148/ bpt6k56149447.item, consulté le 17 août 2020.

Campos, Rémy (1995), «'Mens sana in corpore sano'. L'introduction de l'histoire de la musique au Conservatoire ", dans Emmanuel Hondré (dir.), Le Conservatoire de Paris, regards sur une institution et son histoire, Paris, Association du bureau des élèves du CNSMDP.

Campos, Rémy (2016), Le Conservatoire de Paris et son histoire. Une institution en question, Paris, L'œil d'or.

Chassain-Dolliou (1995), Le Conservatoire de Paris ou les voies de la création, Paris, Gallimard.

Cité de la musique - Philharmonie de Paris, " Métiers de la musique ", https://metiers. philharmoniedeparis.fr/, consulté le 23 octobre 2019.

Conservatoire National Supérieur de musique et de danse de Paris, www.conservatoiredeparis.fr, consulté en février 2019.

Ministère de la Culture (2016), «Bulletin officiel no 261 (août 2016) », www.culture.gouv.fr/Espacedocumentation/Bulletin-officiel/Bulletin-officiel-n-261-aout-2016, consulté le 10 juillet 2020.

Pébrier, Sylvie (2015), Étude relative à la musique classique et ses publics, Ministère de la culture et de la communication, Paris, Direction générale de la création artistique, Service de l'inspection et de la création artistique, Rapport $\mathrm{n}^{\circ}$ SIE 2015 35, février, www.culture.gouv.fr/content/ download/125443/file/2015 rap pebrier musique publics.p df?inLanguage=fre-FR, consulté le 10 juillet 2020 .

Ravet, Hyacinthe (2011), Musiciennes. Enquête sur les femmes et la musique, Paris, Autrement.

Schneider, Corinne (2000), L'enseignement de la culture musicale dans les conservatoires, Paris, Cité de la musique, département pédagogie et documentation musicales. 